\title{
BiHamiltonian Formulations of the Bateman Equation
}

\author{
J.A. Mulvey \\ Department of Mathematical Sciences \\ University of Durham, \\ South Road, \\ DURHAM, DH1 3LE, UK \\ email: J.A.Mulvey@durham.ac.uk \\ 21 September 1995
}

\section{Preprint DTP $/ 95 / 51$}

\begin{abstract}
We discuss a class of evolution equations equivalent to the simplest Universal Field Equation, the so-called Bateman equation, and show that all of them possess (at least) biHamiltonian structure. The first few conserved charges are calculated.
\end{abstract}




\section{Introduction}

Bateman's equation arose in a 1929 paper on hydrodynamics [1] when he studied the properties of the equation:

$$
\Delta=\phi_{x}^{2} \phi_{t t}-2 \phi_{x} \phi_{t} \phi_{x t}+\phi_{t}^{2} \phi_{x x}=0 .
$$

Here $\phi$ is a scalar field and subscripts denote partial derivatives. Many of the interesting properties of the Bateman equation are dealt with in [2]; its generalisations to higher dimensions are discussed in that and subsequent papers [3, 因 5], where they become the Universal Field Equations, a class of partial differential equations with intriguing Lagrangian formulations. Further analyis appears in [6]. In the present case, we find that (1.1) has an infinite

number of inequivalent Lagrangians. Any smooth, real-valued function homogeneous of degree one in the derivatives $\phi_{x}$ and $\phi_{t}$, and with arbitrary dependence on $\phi$, works as a Lagrangian for $\Delta$.

This is straightforward to prove. Suppose $\mathcal{L}\left(\phi, \phi_{x}, \phi_{t}\right)$ is a candidate Lagrangian. By Euler's theorem on homogeneous functions:

$$
\phi_{x} \frac{\partial \mathcal{L}}{\partial \phi_{x}}+\phi_{t} \frac{\partial \mathcal{L}}{\partial \phi_{t}}=\mathcal{L}
$$

It follows easily that:

$$
\begin{gathered}
\phi_{x} \frac{\partial^{2} \mathcal{L}}{\partial \phi_{x}^{2}}+\phi_{t} \frac{\partial^{2} \mathcal{L}}{\partial \phi_{x} \partial \phi_{t}}=0, \\
\phi_{x} \frac{\partial^{2} \mathcal{L}}{\partial \phi_{x} \partial \phi_{t}}+\phi_{t} \frac{\partial^{2} \mathcal{L}}{\partial \phi_{t}^{2}}=0 \\
\phi_{x} \frac{\partial^{2} \mathcal{L}}{\partial \phi \partial \phi_{x}}+\phi_{t} \frac{\partial^{2} \mathcal{L}}{\partial \phi \partial \phi_{t}}=\frac{\partial \mathcal{L}}{\partial \phi} .
\end{gathered}
$$

Now, if we expand the Euler-Lagrange expression,

$$
\mathcal{E} \mathcal{L}=\frac{\partial \mathcal{L}}{\partial \phi}-D_{x}\left(\frac{\partial \mathcal{L}}{\partial \phi_{x}}\right)-D_{t}\left(\frac{\partial \mathcal{L}}{\partial \phi_{t}}\right),
$$

and use the homogeneity properties (1.3) the equation of motion becomes:

$$
\mathcal{E} \mathcal{L}=\frac{1}{\phi_{x} \phi_{t}} \frac{\partial^{2} \mathcal{L}}{\partial \phi_{x} \partial \phi_{t}}\left(\phi_{x}^{2} \phi_{t t}-2 \phi_{x} \phi_{t} \phi_{x t}+\phi_{t}^{2} \phi_{x x}\right) .
$$


This is equivalent to (1.1) on extremals of $\mathcal{L}$.

Note that the homogeneous equations in (1.3) imply that $\mathcal{L}$ is always singular. Also, notice that if there is no $\phi$ dependence in the Lagrangian, the Euler-Lagrange equation is in the form of a conserved current, and so there are an infinite number of conservation laws for (1.1). We will use this observation to construct Hamiltonian conservation laws that are properly in involution.

It should be noted that much is already known about the Bateman equation from the classical theory of partial differential equations. For example, in common with other quasilinear second-order equations [7], $\Delta$ can be linearised by a Legendre transform [5]. With the change of variables,

$$
\begin{aligned}
\xi & =\phi_{x} \\
\eta & =\phi_{t} \\
\omega(\xi, \eta) & =x \xi+y \eta-\phi,
\end{aligned}
$$

(1.1) is equivalent to:

$$
\xi^{2} \omega_{\xi \xi}+2 \xi \eta \omega_{\xi \eta}+\eta^{2} \omega_{\eta \eta}
$$

This linearised equation supplies an implicit solution for $\Delta=0$. According to a theorem of Kumei and Bluman [8], the linearisability property is due to the existence of a first-order generalised symmetry of (1.1) whose characteristic $Q$ depends on an arbitrary solution of the linear equation:

$$
\phi_{x}^{2} \frac{\partial^{2} Q}{\partial \phi_{x}^{2}}+2 \phi_{x} \phi_{t} \frac{\partial^{2} Q}{\partial \phi_{x} \partial \phi_{t}}+\phi_{t}^{2} \frac{\partial^{2} Q}{\partial \phi_{t}^{2}}=0 .
$$

From this we deduce that any $Q$ homogeneous of degree zero or one in the first derivatives of $\phi$ is a characteristic for a generalised symmetry of (1.1).

The task at hand is to study a more formal matter, namely whether there is a Hamiltonian structure or structures associated with the various Lagrangian formulations of the Bateman equation, and how its integrability features in such a formalism. The basic reference for the techniques used is [9]. 


\section{Hamiltonian Analysis}

The Hamiltonian and multi-Hamiltonian descriptions of the Born-Infeld equation have already been studied by Nutku and various collaborators [10, 11] and the aim here is to perform a similar analysis of the Bateman equation. The results of these papers can be adapted to the present case simply by using the $\lambda=0$ limit of the (unique) Born-Infeld Lagrangian,

$$
\mathcal{L}_{B I}=\sqrt{\lambda+\phi_{x}^{2}-\phi_{t}^{2}}
$$

This essentially repeats the work in [1] but, as we have seen, the set of admissible Lagrangians for (1.1) is far larger than this. We can discuss Hamiltonian formulations corresponding to a large class of these non-standard Bateman Lagrangians and in general we will find behaviour quite different from the Born-Infeld case.

We need to find an evolution equation (or system of evolution equations) of the form,

$$
\mathbf{u}_{t}=\mathcal{J} \delta \mathcal{H}[\mathbf{u}]
$$

where $\mathcal{H}[\mathbf{u}]$ is the Hamiltonian functional of the dependent variables $u, v$ and $\mathcal{J}$ is a skewadjoint differential operator. In this context, "skew-adjoint" means that

$$
\int_{\Omega} A \cdot \mathcal{J} B d x=\int_{\Omega} B \cdot \mathcal{J}^{*} A d x=-\int_{\Omega} B \cdot \mathcal{J} A d x
$$

assuming that the support of $A$ and $B$ on the region $\Omega$ allows formal integration by parts. The associated Poisson bracket is:

$$
\{\mathcal{P}, \mathcal{Q}\}=\int \delta \mathcal{P} \cdot \mathcal{J} \delta \mathcal{Q} d x
$$

This bracket must satisfy the Jacobi identity, which can be verified using Olver's convenient method using functional tri-vectors [12, 9]. We require that this system is equivalent to (1.1) under some suitable change of variables.

Ideally, we would like to be able to find two such structures for our evolution equations. Then, provided some compatibility criteria are satisfied, Magri's theorem [13] guarantees the complete integrability of the equation and allows construction of the Lax pair representation and the associated conservation laws. 
The most direct approach is suggested by the fact mentioned earlier, that if the Bateman Lagrangian has no $\phi$ dependence, (1.1) can be expressed as a conservation law:

$$
D_{x}\left(\frac{\partial \mathcal{L}}{\partial \phi_{x}}\right)+D_{t}\left(\frac{\partial \mathcal{L}}{\partial \phi_{t}}\right)=0 .
$$

The biHamiltonian stucture of equations expressible in the form of a conservation law was demonstrated by Nutku [14]. As mentioned above, there are an infinite number of ways of expressing the Bateman equation as a divergence-free current, and there is a biHamiltonian structure associated with each.

Following [14], the divergence (2.5) may be rewritten as the smoothness condition for a function $\psi$.

$$
\begin{aligned}
& \psi_{x}=\frac{\partial \mathcal{L}}{\partial \phi_{t}} \\
& \psi_{t}=\frac{\partial \mathcal{L}}{\partial \phi_{x}}
\end{aligned}
$$

In accordance with the standard Hamiltonian technique, the field $\phi$ is mapped to a pair of variables $u, v$, where

$$
\begin{aligned}
& u=\phi_{x}, \\
& v=\psi_{x}
\end{aligned}
$$

The $t$-derivatives can then be expressed in terms of these new variables:

$$
\begin{aligned}
\phi_{t} & =U(u, v), \\
\psi_{t} & =V(u, v) .
\end{aligned}
$$

Consequently, the equation of motion and the smoothness condition for $\phi$ can be summarised by the condition of closure for the pair of exact 1-forms,

$$
\begin{aligned}
& d \phi=u d x+U d t, \\
& d \psi=v d x+V d t,
\end{aligned}
$$


namely that:

$$
\begin{aligned}
& u_{t}-D_{x}(U)=0 \\
& v_{t}-D_{x}(V)=0 .
\end{aligned}
$$

Given our assumptions about the nature of the Lagrangian, we can deduce the form of $U$ and $V$. From (1.3), we know that the derivatives of $\mathcal{L}$ with respect to $\phi_{x}$ and $\phi_{t}$ are homogeneous of degree zero. So, let us write (2.6) as,

$$
\begin{aligned}
& \psi_{x}=F\left(\frac{\phi_{t}}{\phi_{x}}\right), \\
& \psi_{t}=G\left(\frac{\phi_{t}}{\phi_{x}}\right),
\end{aligned}
$$

where $F$ and $G$ are smooth, invertible functions. Solving the first of these relations for $\phi_{t}$ tells us that,

$$
U(u, v)=u F^{-1}(v),
$$

and substitution into the second,

$$
V(u, v)=G\left(F^{-1}(v)\right) .
$$

At this point, we attempt to impose the Hamiltonian structure. A nice result about this type of equation [14] is that it admits the canonical, "flat" Poisson structure defined by the structure matrix,

$$
\mathcal{J}_{1}=\left(\begin{array}{cc}
0 & D_{x} \\
D_{x} & 0
\end{array}\right),
$$

and a Hamiltonian $\mathcal{H}_{1}$ dependent only on $u$ and $v$, subject to the conditions,

$$
\frac{\partial \mathcal{H}_{1}}{\partial u}=-V, \frac{\partial \mathcal{H}_{1}}{\partial v}=-U,
$$

which in turn impose compatibility criteria on $U$ and $V$.

In this example, the compatibility requires that:

$$
G^{\prime}\left(F^{-1}(v)\right) \frac{\partial}{\partial v}\left(F^{-1}(v)\right)=F^{-1}(v) .
$$


Bearing this in mind, we end up with a general Hamiltonian framework for the Bateman equation. The Hamilton equations are easily seen to reduce to the form,

$$
\begin{aligned}
& u_{t}=D_{x}\left(u F^{-1}(v)\right), \\
& v_{t}=F^{-1}(v) v_{x} .
\end{aligned}
$$

The structure matrix is $\mathcal{J}_{1}$ and the Hamiltonian is:

$$
\mathcal{H}_{1}=u G\left(F^{-1}(v)\right)
$$

There is also a biHamiltonian structure associated with these equations. For this "conserved current" type of system there is always a conserved quantity of the form [14],

$$
\mathcal{H}_{0}=u v .
$$

Following the standard program for biHamiltonian systems [9], we postulate this as a conserved Hamiltonian which reproduces the equations of motion when used with a second structure matrix $\mathcal{J}_{2}$ :

$$
\mathcal{J}_{1} \delta \mathcal{H}_{1}=\mathcal{J}_{2} \delta \mathcal{H}_{0}
$$

Provided this second structure is "compatible" with the first, meaning that any linear combination of the two is also a Poisson structure, this construction will give rise to a (hopefully infinite) sequence of conserved quantities $\mathcal{H}_{k}$ such that,

$$
\mathcal{J}_{1} \delta \mathcal{H}_{k}=\mathcal{J}_{2} \delta \mathcal{H}_{k-1}
$$

which can be generated by the recursion operator:

$$
\mathcal{R}=\mathcal{J}_{2} \mathcal{J}_{1}^{-1} .
$$

We know that the general form of $\mathcal{J}_{2}$, as the most general first-order, skew-adjoint, matrix differential operator is,

$$
\mathcal{J}_{2}=\left(\begin{array}{cc}
2 m D_{x}+m_{x} & 2 p D_{x}+(p+q)_{x} \\
2 p D_{x}+(p-q)_{x} & 2 n D_{x}+n_{x}
\end{array}\right),
$$


where $m, n, p, q$ are functions of $u$ and $v$. By using the condition (2.20) and demanding that it reproduces (2.17), restrictions on the form of $\mathcal{J}_{2}$ can be found. Nutku 14 proves that these restrictions also ensure the closure of the Jacobi identity.

It is difficult to write down a convenient general form for the most general structure so derived, but a useful simplifying assumption is to take $n=0$. Then we find a possible candidate for the second structure given by,

$$
\begin{aligned}
m & =\frac{k}{v^{2}}, \\
n & =0, \\
p & =\frac{1}{2} F^{-1}(v), \\
q & =\frac{1}{2} F^{-1}(v)+k^{\prime},
\end{aligned}
$$

where $k$ and $k^{\prime}$ are constants. It is straightforward to ascertain that this structure is compatible with the $\mathcal{J}_{1}$ : the combination $\mathcal{J}_{1}+\mathcal{J}_{2}$ is skew adjoint and satisfies the Jacobi identity. So $\mathcal{J}_{2}$ can be used to find a second conserved Hamiltonian and thence a whole hierarchy of conserved quantities.

The associated recursion operator is the pseudodifferential operator,

$$
\mathcal{R}=\mathcal{J}_{2} \mathcal{J}_{1}^{-1}=\left(\begin{array}{cc}
F^{-1}(v)+\frac{\partial}{\partial v}\left(F^{-1}\right) v_{x} D_{x}^{-1} & 2 \frac{k}{v^{2}}-2 \frac{k}{v^{3}} v_{x} D_{x}^{-1} \\
0 & F^{-1}(v)
\end{array}\right)
$$

using the values of $p, q$, and $m$ in (2.24).

It is known [9] that a system of evolution equations of the form,

$$
\mathbf{u}_{t}=\mathbf{K}[\mathbf{u}]
$$

which admits a recursion operator, admits a Lax-type representation,

$$
\mathrm{L}_{t}=[\mathcal{A}, \mathrm{L}]
$$

if the $\mathrm{L}$ is the recursion operator $(2.25)$ and $\mathcal{A}$ is the Fréchet derivative:

$$
\mathcal{A}=\mathrm{D}_{\mathbf{K}}=\left.\left(\frac{d}{d \varepsilon} \mathbf{K}\left[\mathbf{u}_{\varepsilon}\right]\right)\right|_{\varepsilon=0}
$$


Here, $\mathbf{u}_{\varepsilon}$ denotes a one-parameter $(\varepsilon)$ family of perturbations of $\mathbf{u}$.

In our example, the Fréchet derivative is:

$$
\mathcal{A}=\left(\begin{array}{cc}
D_{x}\left(F^{-1}(v) \cdot\right) & D_{x}\left(u \frac{\partial}{\partial v} F^{-1}(v) \cdot\right) \\
0 & D_{x}\left(F^{-1}(v) \cdot\right)
\end{array}\right) .
$$

The argument of the $\mathcal{A}$ should be inserted as indicated by the dots. Substitution of (2.29) and (2.25) into (2.27) reproduces the Hamilton equations (2.17).

Now, using the recurrence (2.21), we can start to calculate conserved quantities. The first conserved Hamiltonian is found by considering:

$$
\mathcal{J}_{2} \delta \mathcal{H}_{1}=\mathcal{J}_{1} \delta \mathcal{H}_{2}
$$

It turns out to be:

$$
\mathcal{H}_{2}=\int^{v}\left\{u\left(F^{-1}(w)\right)^{2}+\frac{2 k}{w^{2}} G\left(F^{-1}(w)\right)\right\} d w .
$$

Continuing the process, we find the third charge:

$$
\mathcal{H}_{3}=\int^{v}\left\{F^{-1}(w)\left(u\left(F^{-1}(w)\right)^{2}+\frac{2 k}{w^{2}} G\left(F^{-1}(w)\right)+\frac{2 k}{w^{2}} \int^{w}\left(F^{-1}\left(w^{\prime}\right)\right)^{2} d w^{\prime}\right)\right\} d w .
$$

The subsequent members of the hierarchy become rapidly more complicated.

It is worth noting that the conserved-current type of equation found by Nutku admits a third independent Hamiltonian structure, compatible with the first two, with $\mathcal{H}_{2}$ as the relevant Hamiltonian density. Also, while the sequence of conserved charges in principle continues ad infinitum, there may be certain particular cases when the sequence terminates or repeats itself after a finite number of steps. See [11] for an example of this behaviour arising in the biHamiltonian hierarchies of the Born-Infeld equation. Essentially the same results can be found for the Bateman case by choosing the Lagrangian (2.1) with $\lambda=0$.

\section{Acknowledgements}

It is a pleasure to thank David Fairlie for his comments and advice. The author is financially supported by the Department of Education for Northern Ireland. 


\section{References}

[1] H. Bateman. Proc. Roy. Soc. London A125 (1929) 598.

[2] D.B. Fairlie, J. Govaerts, and A. Morozov. Nucl. Phys. B373 (1992) 214.

[3] D.B. Fairlie and J. Govaerts. Phys. Lett. 281B (1992) 49.

[4] D.B. Fairlie and J. Govaerts. J. Math. Phys. 33 (1992) 3543.

[5] D.B. Fairlie and J. Govaerts. J. Phys. A 26 (1993) 3339.

[6] D.R. Grigore. On equations with universal invariance. (Preprint $\backslash$ protect \vrule width0pt \protect \href\{http://arxiv.org/abs/hep-th/9410195\}\{hep-th/941 (1994).

[7] R Courant and D Hilbert. Methods of Mathematical Physics, Volume II. (Interscience, New York, 1962).

[8] S. Kumei and G. Bluman. SIAM J. Appl. Math. 42 (1982) 1157.

[9] P.J. Olver. Application of Lie Groups to Differential Equations, (2nd ed). (SpringerVerlag, New York, 1993).

[10] Y. Nutku and P.J. Olver. J. Math. Phys. 29 (1988) 1610.

[11] M. Arik, F. Neyzi, Y. Nutku, P.J. Olver, and J. Verosky. J. Math. Phys. 30 (1989) 1138.

[12] P.J. Olver. Math. Proc. Camb. Phil. Soc. 88 (1980) 71.

[13] G. Magri. J. Math. Phys. 19 (1978) 1156.

[14] Y. Nutku. J. Math. Phys. 26 (1985) 1237. 